\title{
LIST OF THE INTERNATIONAL REVIEWERS IN 2017
}

The Korean Journal of Orthodontics enjoyed another great successful year in 2017. Hundreds of well-prepared manuscripts have been submitted from many countries for publication and, in reviewing of those manuscripts, our orthodontic experts have contributed in various ways to maintain a higher standard of the Journal. Among them, we hopes to extend our special thanks to the following international reviewers invited in 2017. Their expertise, dedication, and uncompromising demand for scientific sophistication were all instrumental to assuring the high quality expected by our readers.

\author{
Adusumilli Sai Prakash, India \\ Ayşe Gulşen, Turkey \\ Baek Il Kim, Korea \\ Bong-Kuen Cha, Korea \\ Carlos Flores-Mir, Canada \\ Cecy Martins Silva, Brazil \\ Cheol-Hyun Moon, Korea \\ Chiarella Sforza, Italy \\ Chihiro Arai, Japan \\ Chooryung Chung, Korea \\ Chung Ho Kau, United States \\ Chung Ju Hwang, Korea \\ Derya Germec Cakan, Turkey \\ Dibart Serge, United States \\ Ding Bai, China \\ Dong Hwa Chung, Korea \\ Dong-Soon Choi, Korea \\ DongYul Lee, Korea \\ Elcin Esenlik, Turkey \\ Ellen BeGole, United States \\ Emre Kayalar, Turkey \\ Eric Liou, Taiwan \\ Eung-Kwon Pae, United States \\ Farhad B. Naini, United Kingdom \\ Fariborz Amini, Iran \\ Flavio Uribe, United States \\ Gaetano Isola, Italy \\ Giulio Alessandri Bonetti, Italy \\ Gundega Jakobsone, Latvia \\ H. W. Fields, United States \\ Hakan Turkkahraman, Turkey \\ Hande Gorucu Coskuner, Turkey \\ Hatice Gokalp, Turkey \\ Hee-Moon Kyung, Korea \\ Heon Jae Cho, Korea \\ Hoi-Jeong Lim, Korea \\ Hooman Zarif Najafi, Iran \\ Hyo-Sang Park, Korea \\ Hyo-Won Ahn, Korea \\ Il-Hyung Yang, Korea \\ Il-Sik Cho, Korea \\ Insan Jang, Korea \\ J. Martin Palomo, United States \\ Jae Hyun Park, United States \\ Jaehoon Lee, Korea
}

Jae-Woo Park, Korea

Jaewook Nam, Korea

Janghoon Ahn, Korea

Jeong-Chae Lee, Korea

Jeong-Ho Choi, Korea

J. H. Calderón, Mexico

Jianhua Hou, China

Jin Hyoung Cho, Korea

Jin-Hyoung Cho, Korea

Jiuxiang Lin, China

Ji-Yeon Lee, Korea

Joe Antoun, New Zealand

Jong-Moon Chae, Korea

Jong-Wan Kim, Korea

Joon Row, Korea

Jung-Yul Cha, Korea

Junji Sugawara, Japan

Kee-Joon Lee, Korea

Khalid H. Zawawi, Saudi Arabia

Ki Beom Kim, United States

Ki-Ho Park, Korea

Krishna Sharma, India

Kwang-Mahn Kim, Korea

Kyung Hwa Kang, Korea

Kyung-Ho Kim, Korea

Kyung-Min Lee, Korea

Leopold Wagner, Poland

Letizia Perillo, Italy

L. J. Lo, Taiwan

L. M. Galantucci, Italy

Lorenzo Franchi, Italy

M. Ali Darendeliler, Australia

Manuel Lagravere, Canada

Masaru Yamaguchi, Japan

M. G. Woods, Australia

Min-Ho Jung, Korea

Minji Kim, Korea

Mohammad Khursheed Alam, Malaysia

Mohd Masood, Malaysia

Nam-Ki Lee, Korea

Nasrin Farhadian, Iran

Na-young Chang, Korea

Neslihan Ebru Şenışık, Turkey

Rafael Scaf de Molon, Brazil
Ravindra Nanda, United States

Renato Bigliazzi, Brazil

RF Viecilli, United States

Rogelio J. Scougall-Vilchis, Mexico

S. Sood, India

Sang Jin Sung, Korea

Sang-Min Lee, Korea

Seniz Karacay, Turkey

Seong Sik Kim, Korea

Seong-Hun Kim, Korea

Seung-Hak Baek, Korea

Seung-Youp Lee, Korea

Siti Adibah Othman, Malaysia

Spyridon N. Papageorgiou, Germany

Sug-Joon Ahn, Korea

Su-Jung Kim, Korea

Sung Jin Kim, Korea

Sung-Hoon Lim, Korea

Sunghwan Choi, Korea

Sung-Nam Gang, Korea

Sung-Seo Mo, Korea

S. W. Herring, United States

Tae Hyun Choi, Korea

Tae-Woo Kim, Korea

Tancan Uysal, Turkey

Tarek H. EL-Bialy, Canada

Tian-Min Xu, China

Visnja Katic, Croatia

Wan Nurazreena Wan Hassan, Malaysia

Weidong Tian, China

Wey Mang Chek, Malaysia

Won Hee Lim, Korea

Woo-Sung Son, Korea

Xiaowei Li, China

Yanheng Zhou, China

Yong-Il Kim, Korea

Yoon Jeong Choi, Korea

Yoon-Ah Kook, Korea

Yoon-Goo Kang, Korea

Yoonji Kim, Korea

Yoon-Ji Kim, Korea

Young Ho Kim, Korea

Young-Guk Park, Korea

Yu-lou Tian, China 